\title{
Impact Pressure Distribution in Flat Fan Nozzles for Descaling Oil Wells
}

\author{
Abubakar Jibrin Abbas, Ghasem Ghavami Nasr, Amir Nourian and Godpower Chimagwu Enyi \\ Spray Research Group, School of Computing, Science and Engineering, University of Salford, Manchester M5 4WT, UK
}

Received: February 15, 2016 / Accepted: March 09, 2016 / Published: June 30, 2016.

\begin{abstract}
The suitability of high pressure nozzles in terms of impact upon targeted surfaces has indicated its effectiveness for the cleaning of oil production tubing scale, which has recently attracted wider industrial applications considering its efficiency, ease of operation and cost benefit. In the oil and gas production, these nozzles are now used for cleaning the scale deposits along the production tubing resulted mainly from salt crystallization due to pressure and temperature drop. Detailed characterizations of flat-fan nozzle in terms of droplet sizes and mean velocities will benefit momentum computations for the axial and radial distribution along the spray width, with the view of finding the best stand-off distance between the target scale and the spray nozzle. While the droplet sizes and the velocities determine the momentum at impact, measuring droplet sizes has been known to be difficult especially in the high density spray region, still laboratory characterization of nozzles provides a reliable data especially avoiding uncontrollable parameters. While several researches consider break up insensitive to the cleaning performance, this research investigates the experimental data obtained using PDA (phase doppler anemometry) which led to established variation in momentum across the spray width thus, non-uniformity of impact distribution. Comparative model was then developed using Ansys Fluent code, which verifies the eroded surfaces of material using the flat-fan atomizer to have shown variability in the extent of impact actions due to kinetic energy difference between the center and edge droplets. The study's findings could be useful in establishing the effect of droplet kinetic energies based on the spray penetration, and will also add significant understanding to the effect of the ligaments and droplets, along the spray penetration in order to ascertain their momentum impact distribution along the targeted surface.
\end{abstract}

Key words: Impact pressure, flat fan nozzle, descaling, oil well, phase doppler anemometry, sprays.

\section{Introduction}

The performance of high pressure nozzles in terms of impact upon targeted surfaces has indicated its suitability for the cleaning of oil production tubing which has recently attracted wide industrial applications considering its efficiency [1-4], ease of operation and attractive cost benefit. In the oil and gas production, these nozzles are now used for cleaning the scale deposits along the production tubing resulted mainly from salt crystallization due to pressure and temperature drop. Detailed characterizations of flat-fan nozzle in terms of droplet sizes and mean velocities will benefit momentum computations for the axial and

Corresponding author: Abubakar Jibrin Abbas, Ph.D., research fields: enhanced oil recovery, flow assurance, natural gas processing, gas hydraulics. radial distribution along the spray width, with the view of finding the best stand-off distance between the target scale and the spray nozzle, while the droplet sizes and the velocities determine the momentum at impact, although measuring droplet sizes has been known to be difficult especially in the high density spray region [5, 6], still laboratory characterization of nozzles provides a reliable data especially as it can avoid uncontrollable parameters [7].

\section{PDA Set-up}

The PDA (phase doppler anemometry) system was set up with the transmitting and receiving optics set to the values shown in Table 1 . The only setting that can be adjusted on the transmitting optics is the power level of the laser. During the experiments, the maximum power setting was used which has the effect 
Table 1 PDA characteristics.

\begin{tabular}{lllll}
\hline & Description & Symbol & Units & Value \\
\hline \multirow{4}{*}{ Transmitting } & Laser power & $P$ & $\mathrm{~mW}$ & 100 \\
optics & Wavelength & $\lambda$ & $\mu \mathrm{m}$ & 514.5 \\
& Beam separation & $d_{f}$ & $\mathrm{~mm}$ & 38 \\
& Focal length & $f_{t r}$ & $\mathrm{~mm}$ & 400 \\
& Beam diameter & $d_{b}$ & $\mathrm{~mm}$ & 5.35 \\
\hline \multirow{2}{*}{ Receiving } & Fringe spacing & $s$ & - & 37 \\
optics & Number of fringes & $N_{f}$ & $\mathrm{~mm}$ & 310 \\
& Focal length & $f_{r c}$ & degree & 72 \\
\hline
\end{tabular}

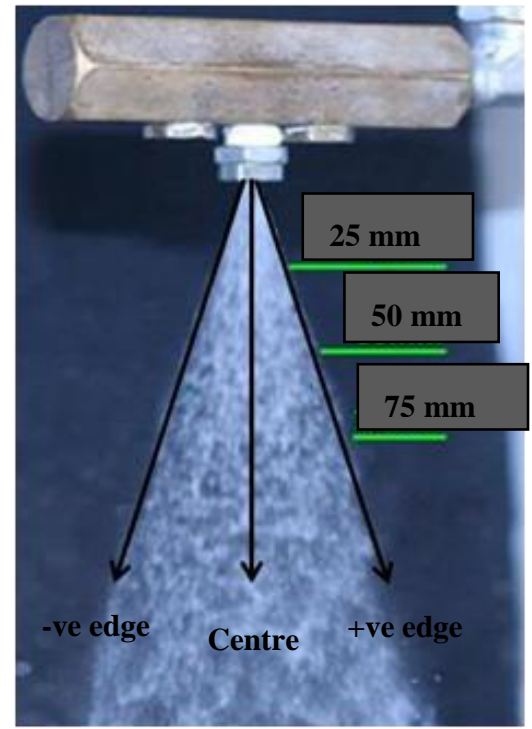

Fig. 1 Measurement grid coordinates.
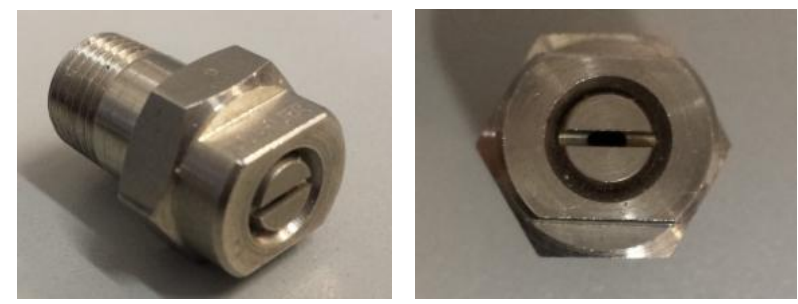

Fig. 2 Flat-fan nozzles.

of increasing the measuring volume.

The receiving optics were set to receive 1 st order refraction from the particles, with the scattering angle being $72^{\circ}$, which is the optimum forward refraction mode with reduced bias in the results due to the reflected light, thus ensuring good scattering light intensity levels (high signal to noise), thus making it suitable for measuring small particles.

The focal length of the receiver was $310 \mathrm{~mm}$.
Decreasing the focal length of the receiver, increases the sensitivity of the optics allowing the receiver to measure smaller particles. However, there are trade-offs with reducing the focal length, such as reducing the size of the measurement volume and reducing the maximum droplet diameter that can be measured. The set focal length of $310 \mathrm{~mm}$ was suitable for measuring the range of particles in the experiments.

\section{Measurement Grid}

The position of the atomizer was centralized about the laser measuring volume, and the atomizer was traversed in the radial plane with reference to the measuring volume (optics fixed). As shown in Fig. 1, droplet size and velocity measurements were taken at $25 \mathrm{~mm}, 50 \mathrm{~mm}$ and $75 \mathrm{~mm}$ at each of the edges, as well as the centre of the atomizer exit for the 0.033 , 0.045 and $0.188 \mathrm{~kg} / \mathrm{s}$ corresponding to $4.8,6.0$ and 10 MPa, respectively or $696.2 \mathrm{psi}, 870.2 \mathrm{psi}$ and 1,450.4 psi, respectively as flow pressures from the flat-fan nozzles shown in Fig. 2. At each down stream location, three radial measurements were taken.

\section{Results and Analysis}

\subsection{Droplet Mean Velocities}

The results obtained for the various runs of the characterization indicated that, although mean velocities generally increase with increasing the liquid loadings $[8,9]$, it is evident that, the droplet at the 
negative and positive edge of the spray width are affected by aerodynamic forces more than the central droplets, which as results experienced fluctuations with subsequent decrease and then increase at $10 \mathrm{MPa}$ $(1,450.4$ psi) as shown in Fig. 3a, and then vice-versa at $4.8 \mathrm{MPa}$ as shown in Fig. 3c instead of expecting a general decrease in velocities as shown in Fig. 3 b. This also confirms why the increase in liquid loadings does not show an appreciable variation between 6.0 $\mathrm{MPa}$ and $10 \mathrm{MPa}$ (870.2 psi and 1,450.4 psi),
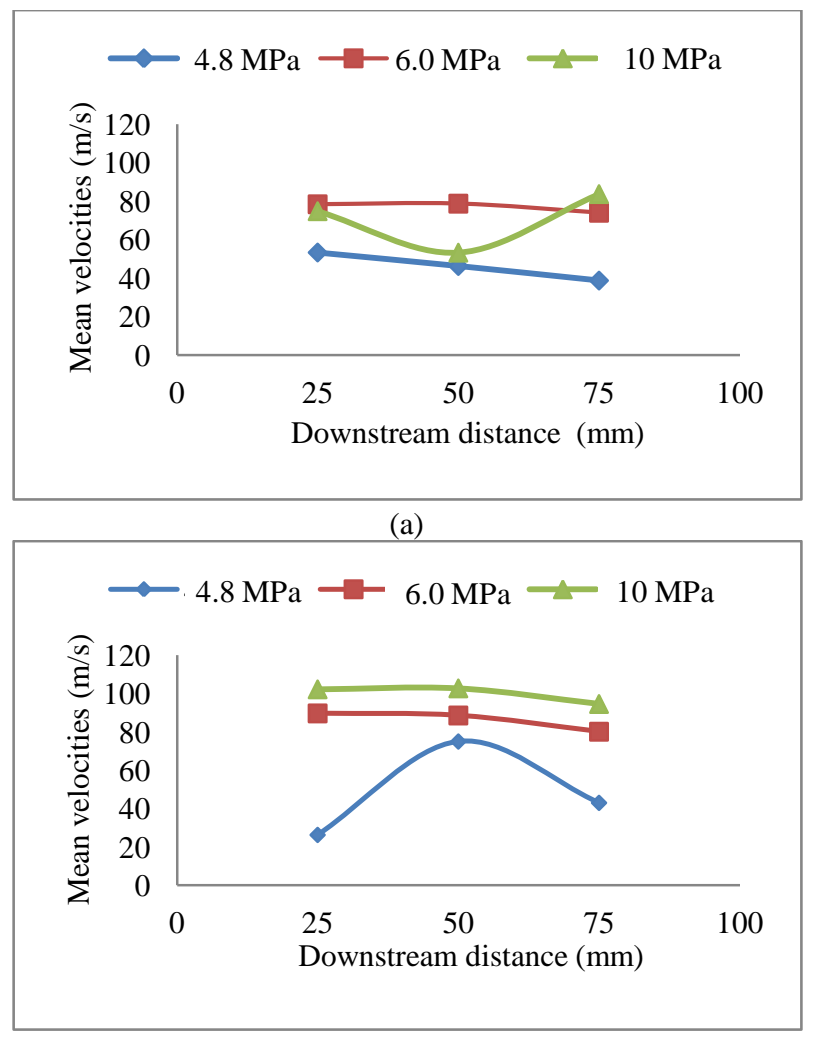

(b)

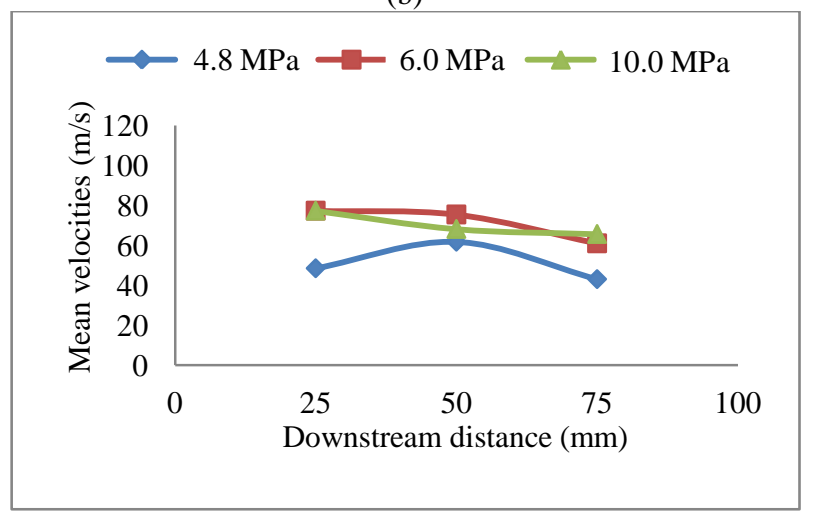

(c)

Fig. 3 Mean velocities at various pressures (a) -ve edge, (b) at centre and (c) +ve edge. especially at the edge of the sprays as shown in Figs. $3 \mathrm{a}$ and $3 \mathrm{c}$.

Indeed, the mean velocities obtained at the centre of the spray seem to follow the expected order of highest velocity at $100 \mathrm{~m} / \mathrm{s}$, then followed by $6 \mathrm{MPa}$ finally 4.8 $\mathrm{MPa}(870.2 \mathrm{psi}$ and $696.2 \mathrm{psi}$ ) as shown in Fig. $3 \mathrm{~b}$. Consequently, higher performance in terms of momentum impact was observed at the centre of the spray with momentum values up to $1.45 \mathrm{e}-5 \mathrm{~kg} \cdot \mathrm{m} / \mathrm{s}$ as the highest obtained.

It is clearly evident that, although spray characterization of flat fan nozzle was known to have lowest flow rate at the centre [10], and highest at the edge, momentum generates from the high droplet velocities at the centre could not be compensated by the increased liquid flow rate at the edge due to lower velocities.

\subsection{Droplet Sizes Measurement}

As part of the efforts to understand the performance of the flat fan nozzle, a corresponding measurement for the SMD (sauterne mean diameters) was recorded using the PDA for the establishment of the actual momentum across the nozzle.

The SMD variations indicate higher consistencies at the beginning of the measurement at $25 \mathrm{~mm}$, with the average droplet sizes at the edge of the spray was able to be highest at $4.8 \mathrm{MPa}$, followed by $6.0 \mathrm{MPa}$ and then $10 \mathrm{MPa}$. However, inconsistencies still exist and the droplet sizes continue to increase at higher pressure with no further atomization's observed as shown in Fig. 4a. However, still the droplet size at the centre of the spray, seems to be less affected by the irregularities (Fig. 4b) compared to the edge SMDs shown in Figs. 4a and 4c.

\subsection{Droplet Momentum}

The results obtained from the PDA measurement based on mean droplet velocities, and SMD were used for the momentum calculations. A droplet of mass (m, $\mathrm{kg})$, travelling with a velocity $(\mathrm{v}, \mathrm{m} / \mathrm{s})$ will have a droplet momentum as: 


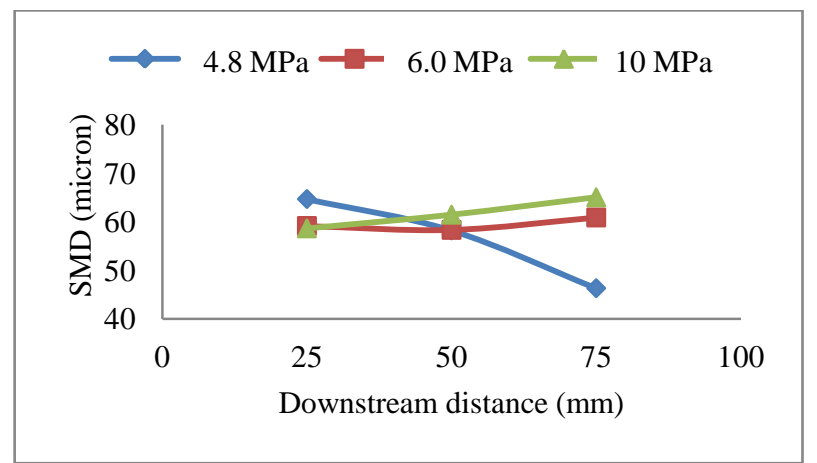

(a)

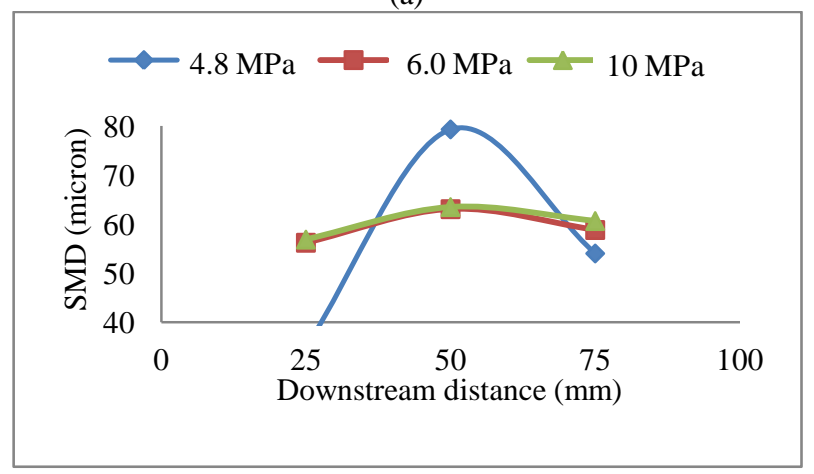

(b)

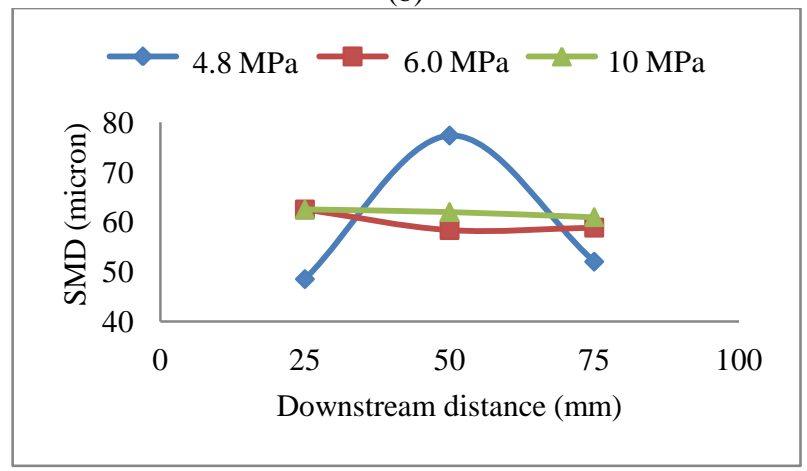

(c)

Fig. 4 Droplet sizes at various pressures (a) -ve edge, (b) at centre and (c) +ve edge.

$$
\text { Droplet momentum }=m \times v \times n
$$

where, $m$ is mass of the droplet, $v$ is velocity, and $n$ is the number of droplet per second.

$$
\begin{aligned}
& m=\rho \times V=\frac{4 \pi \rho}{3}\left(\frac{S M D}{2}\right)^{3} \\
& n=\frac{\text { Droplet capture }}{\text { Time taken }}
\end{aligned}
$$

For 20,000 droplet captured over a period of $20 \mathrm{~s}$, the number of droplet per time equals to 1,000 .

Droplet momentum $=0.5236 \rho \cdot \mathrm{SMD}^{3}$

The calculated was performed as per Eq. (4) and shown in Fig. 5.

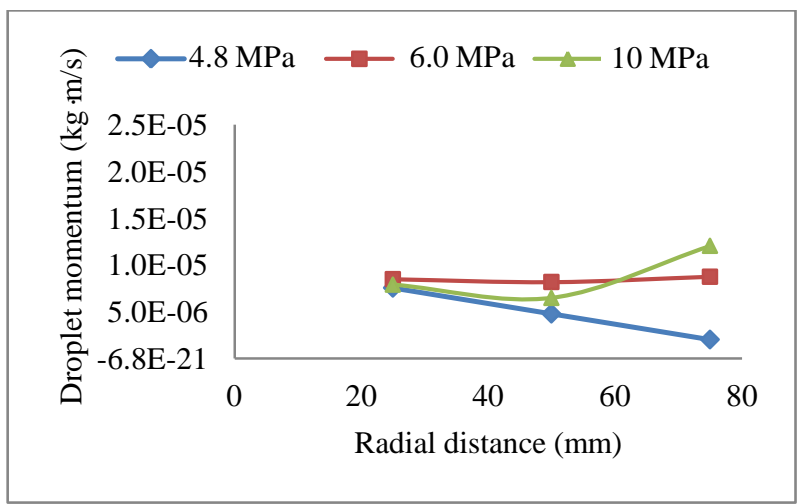

(a)

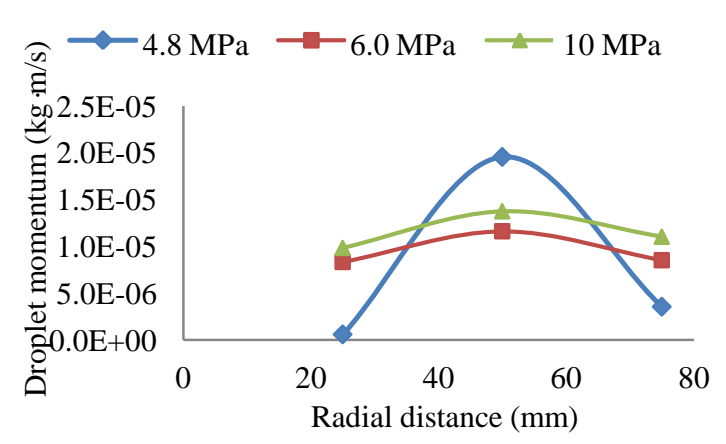

(b)

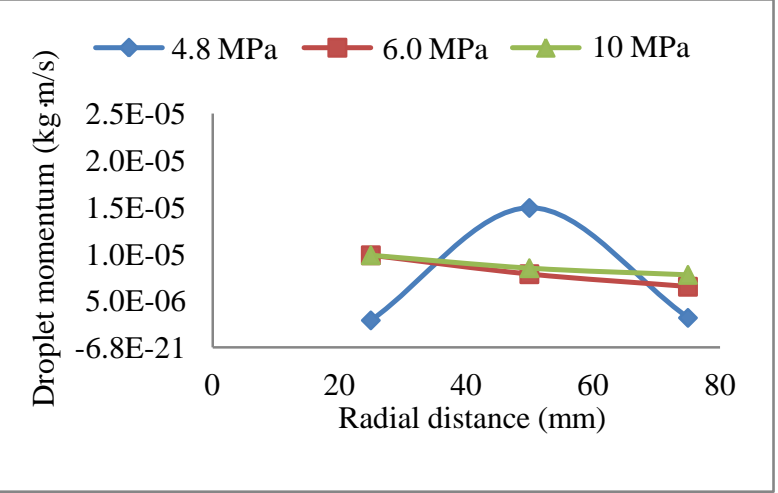

(c)

Fig. 5 Droplet momentums at various positions pressures (a) -ve edge, (b) at centre and (c) +ve edge.

The droplet momentum has indicated higher values at the centre of the spray, compared to the edge where significant reduction can be observed as shown in Figs. $5 \mathrm{a}-5 \mathrm{c}$.

However, additional comparison of the momentum was conducted at the same pressure (liquid loading). The momentum of the droplets computed seems highest at the centre of the spray, at all pressures regardless of the radial and axial positions as shown in Fig. 6. 


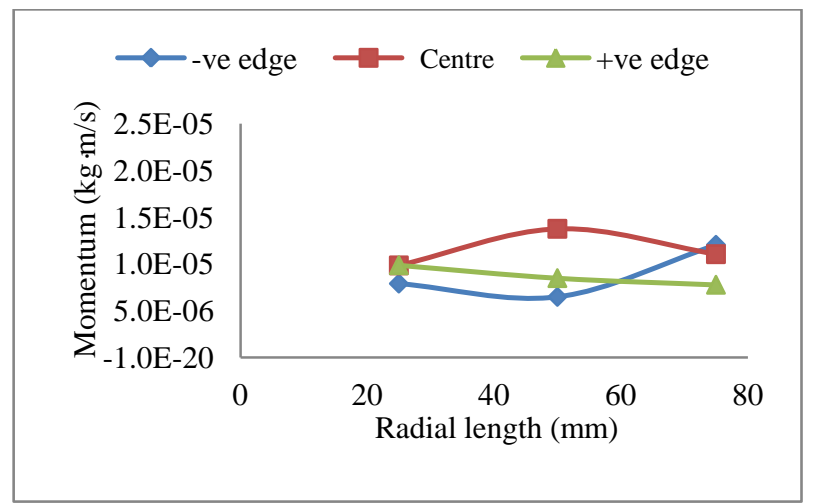

(a)

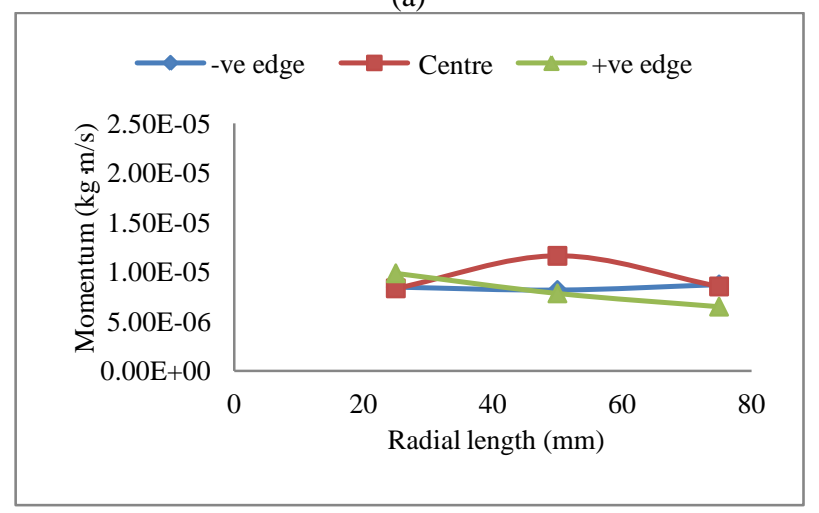

(b)

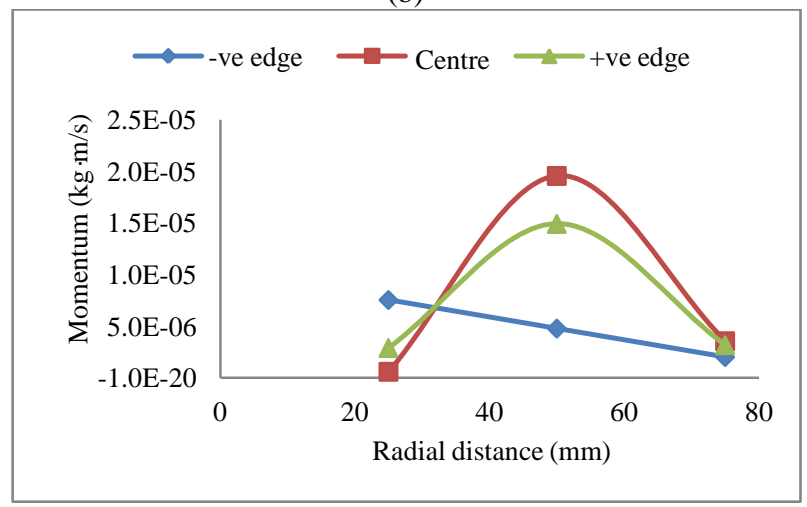

(c)

Fig. 6 Droplet momentums at various pressures (a) 10 MPa, (b) $6 \mathrm{MPa}$ and (c) 4.8 MPa.

\section{Numerical Comparison}

A turbulent flow coming from the exit of a flat-fan nozzle was modelled using Fluent code, with a targeted scale surface to view the impact profile. The distribution of the impact in terms of erosion was seen to be at maximum at the centre of the surface, and decreased along the width of the spray as shown in Fig. 7. It is evident that, the distribution of the impact is non-uniform across the spray width.

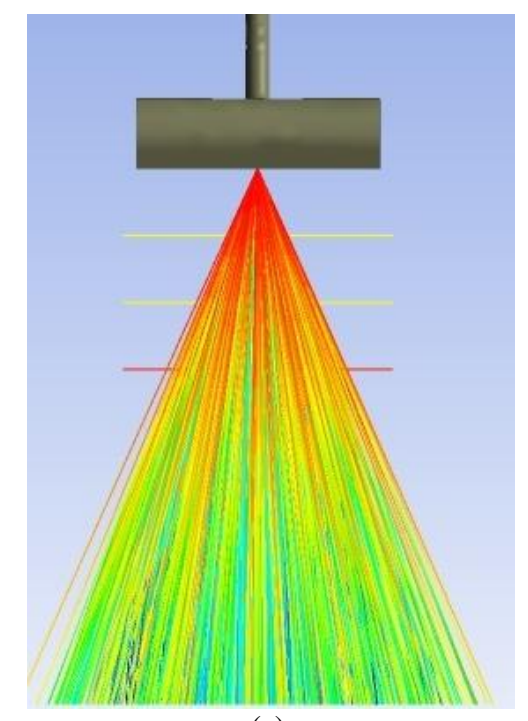

(a)

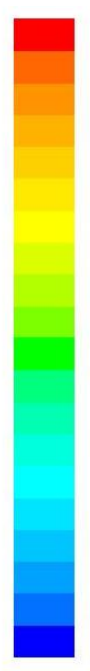

$$
\begin{aligned}
& 1.84 \mathrm{e}-06 \\
& 1.75 \mathrm{e}-06 \\
& 1.66 \mathrm{e}-06 \\
& 1.57 \mathrm{e}-06 \\
& 1.47 \mathrm{e}-06 \\
& 1.38 \mathrm{e}-06 \\
& 1.29 \mathrm{e}-06 \\
& 1.20 \mathrm{e}-06 \\
& 1.11 \mathrm{e}-06 \\
& 1.01 \mathrm{e}-06 \\
& 9.21 \mathrm{e}-07 \\
& 8.29 \mathrm{e}-07 \\
& 7.37 \mathrm{e}-07 \\
& 6.45 \mathrm{e}-07 \\
& 5.53 \mathrm{e}-07 \\
& 4.60 \mathrm{e}-07 \\
& 3.68 \mathrm{e}-07 \\
& 2.76 \mathrm{e}-07 \\
& 1.84 \mathrm{e}-07 \\
& 9.21 \mathrm{e}-08
\end{aligned}
$$$$
0.00 \mathrm{e}+00
$$

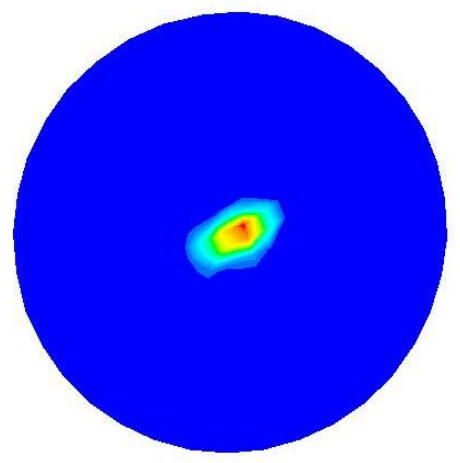

(b)

Fig. 7 Fluent models for droplet impact (a) spray profile, (b) impact area on surface.

\section{Conclusions}

This investigation has adopted the PDA measured droplet sizes, and mean velocities for the establishment of non-uniformity of impact along targeted surface, when using a flat-fan atomizer for high pressure cleaning applications. It is evident that, high droplet velocities along the spray centre impacted higher momentum compared to the edge despite the high liquid distributions.

\section{Acknowledgements}

The authors wish to appreciate the support of SRG 
(Spray Research Group), University of Salford, UK, for the technical and equipment support, and also the PTDF (Petroleum Technology Development Fund), Nigeria, for the sponsorship.

\section{References}

[1] Sangiah, D. K., and Ganippa, L. C. 2010. "Application of Spray Impingement Technique for Characterisation of High Pressure Sprays from Multi-hole Diesel Nozzles." International Journal of Thermal Sciences 49 (2): 409-17.

[2] Payri, R., Garcia, J., Salvador, F., and Gimeno, J. 2005. "Using Spray Momentum Flux Measurements to Understand the Influence of Diesel Nozzle Geometry on Spray Characteristics." Fuel 84 (5): 551-61.

[3] Schlumberger. 2014. Jet Blaster. Report Retrieved from http://www.slb.com/ /media/Files/stimulation/product_sh eets/sandstone/jetblaster_ps.pdf.

[4] Malaguti, S., Fontanesi, S., Cantore, G., Montanaro, A., and Allocca, L. 2013. "Modelling of Primary Breakup Process of a Gasoline Direct Engine Multi-hole Spray." Atomization and Sprays 23 (10): 861-88.

[5] Kohnen, B. T., Pieloth, D., Musemic, E., and Walzel, P.
2011. "Characterization of Full Cone Nozzles." Atomization and Sprays 21 (4): 317-25.

[6] Song, J., Ramasubramanian, C., and Lee, J. G. 2014. "Response of Liquid Jet to Modulated Crossflow." Atomization and Sprays 24 (2): 129-54.

[7] Guler, H., Zhu, H., Ozkan, H. E., and Ling, P. 2012. "Characterization of Hydraulic Nozzles for Droplet Size and Spray Coverage." Atomization and Sprays 22 (8): 627-45.

[8] Manin, J., Bardi, M., Pickett, L. M., and Payri, R. 2015. "Boundary Condition and Fuel Composition Effects on Injection Processes of Diesel Sprays at the Microscopic Level." International Journal of Multiphase Flow 83 (December): 1-35.

[9] Gopireddy, S. R., and Gutheil, E. 2013. "Numerical Investigation of PVP/Water Spray Flows in an Eulerian Framework." In Proceedings of the 25th European Conference on Liquid Atomization and Spray Systems, 412-7.

[10] Bendig, L., Raudenský, M., and Horský, J. 2001. "Descaling with High Pressure Nozzles." In Proceedings of the 17th European Conference on Liquid Atomization and Spray Systems, 742-7. 